\title{
Fundamental Diagrams for Specific Very High Density Crowds
}

\author{
Rainald Löhner ${ }^{1}$. Britto Muhamad ${ }^{2}$. Prabhu Dambalmath ${ }^{2}$. \\ Eberhard Haug ${ }^{2}$ \\ ${ }^{1}$ Center for Computational Fluid Dynamics, George Mason University, Fairfax, USA, \\ E-mail: rlohner@gmu.edu \\ 2 SL Rasch, Oberaichen, Germany, \\ E-mail: britto.muhamad@sl-rasch.de,haugfmly@club-internet.fr
}

Received: 3 August 2017 / Accepted: 19 December 2017

DOI: $10.17815 / \mathrm{CD} .2017 .13$

\begin{abstract}
An experimental campaign was undertaken to measure the pedestrian flow in the region close to the Kaaba during the Hajj pilgrimages of 2014 and 2015. High resolution video and photographs were used. The space was divided into areas of $10 \mathrm{~m}^{2}$. The pedestrians were counted, and the velocity measured from video clips. The results were surprising: the velocity in the very high density region increases, which implies also an increase of the flux. The flux in this region (with more than $8 \mathrm{p} / \mathrm{m}^{2}$ ) reaches values that exceed $3.5 \mathrm{p} / \mathrm{m} / \mathrm{s}$, much higher than previously recorded under more 'standard conditions' in corridors and passages.
\end{abstract}

Keywords Pedestrians $\cdot$ dense crowds $\cdot$ field observations $\cdot$ fundamental diagram

\section{Introduction}

A common experience of daily life is the slow-down of pedestrian and car traffic with higher density. It is therefore not surprising that ever since traffic was measured, this relation of velocity versus density was one of the key findings reported. The intrinsic assumption (or hope) was that there exists some form of general diagram or curve that relates density and velocity, and to this day this diagram has been given the name of 'fundamental diagram' or 'universal diagram'. The fundamental diagram has been the focus of research for several decades. [1,3, 4, 6, 7, 9, 10] and many others (e.g. [2, 5, 8]) have measured and compiled this type of data. While many data points exist for low densities, the very high end regime of densities above $6 \mathrm{p} / \mathrm{m}^{2}$ is very sparse. This is not surprising, as it is difficult (and sometimes dangerous) to set up experiments or perform 


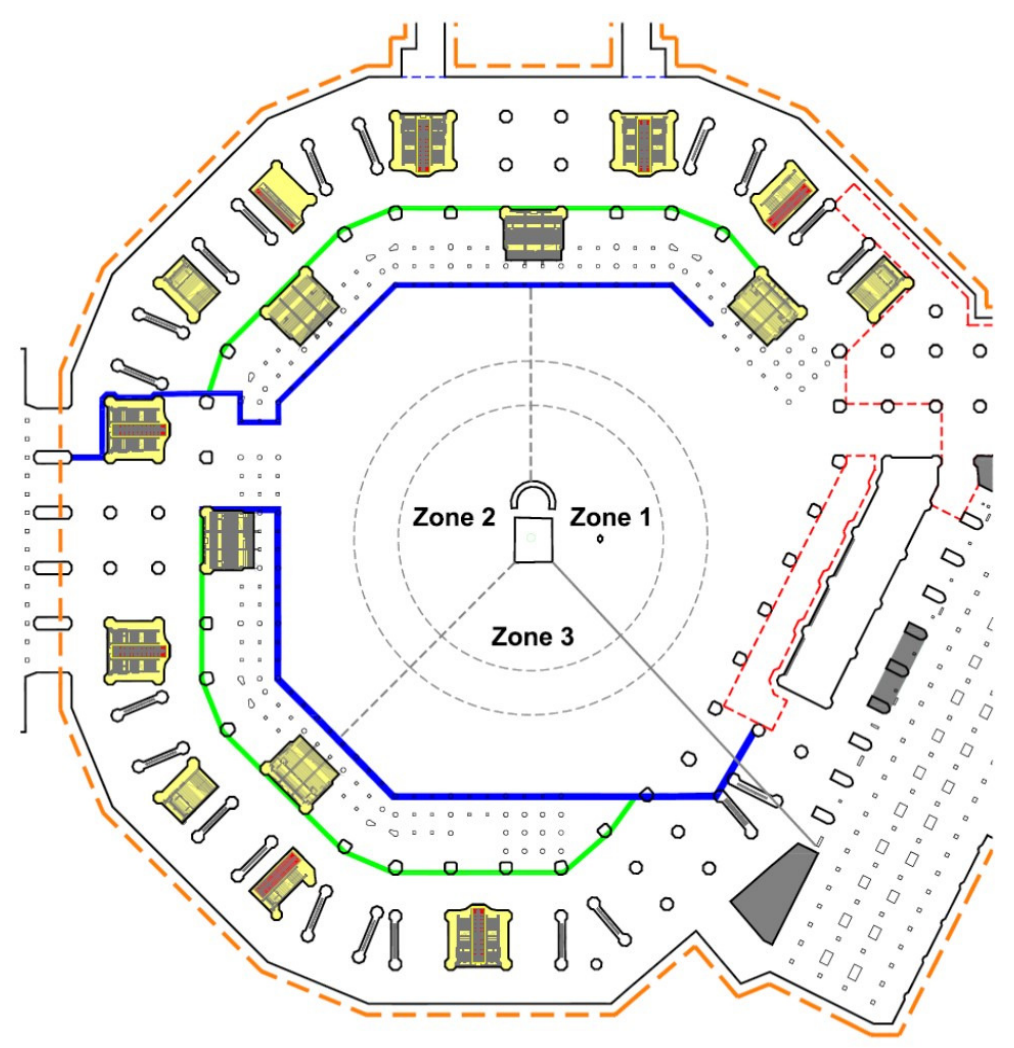

Figure 1 Floor plan of observation area

field observations in this regime. Yet these high densities exist in many crowds, and represent critical elements for both design and operation.

The annual Hajj pilgrimage offers the opportunity to measure high density crowds. Therefore the decision was made to measure the pilgrim flow in the region close to the Kaaba during the annual Hajj pilgrimage. The findings contributed hard data to the regime of very high densities (above $6 \mathrm{p} / \mathrm{m}^{2}$ ). At the same time these results were found to differ from often reported fundamental diagrams, questioning their 'universality' at high density. A preliminary conclusion is that the high density regions strongly depend on the type of motion, such as 'concerted', 'self-organized', 'intention-based', 'marching columns', etc.

\section{Measurement Campaigns}

Measurement campaigns were undertaken during the Hajj pilgrimages of 2014 and 2015. The pilgrims perform the so-called Tawaf by circumambulating the Kaaba at the center of the Sahn seven times. Some pilgrims will stop to touch the black stone at one of the corners of the Kaaba, and others will stop at the Kaaba and offer prayers and supplications while touching it.

High resolution cameras (Canon EOS 5D Mark III with 22 Megapixel resolution) were 

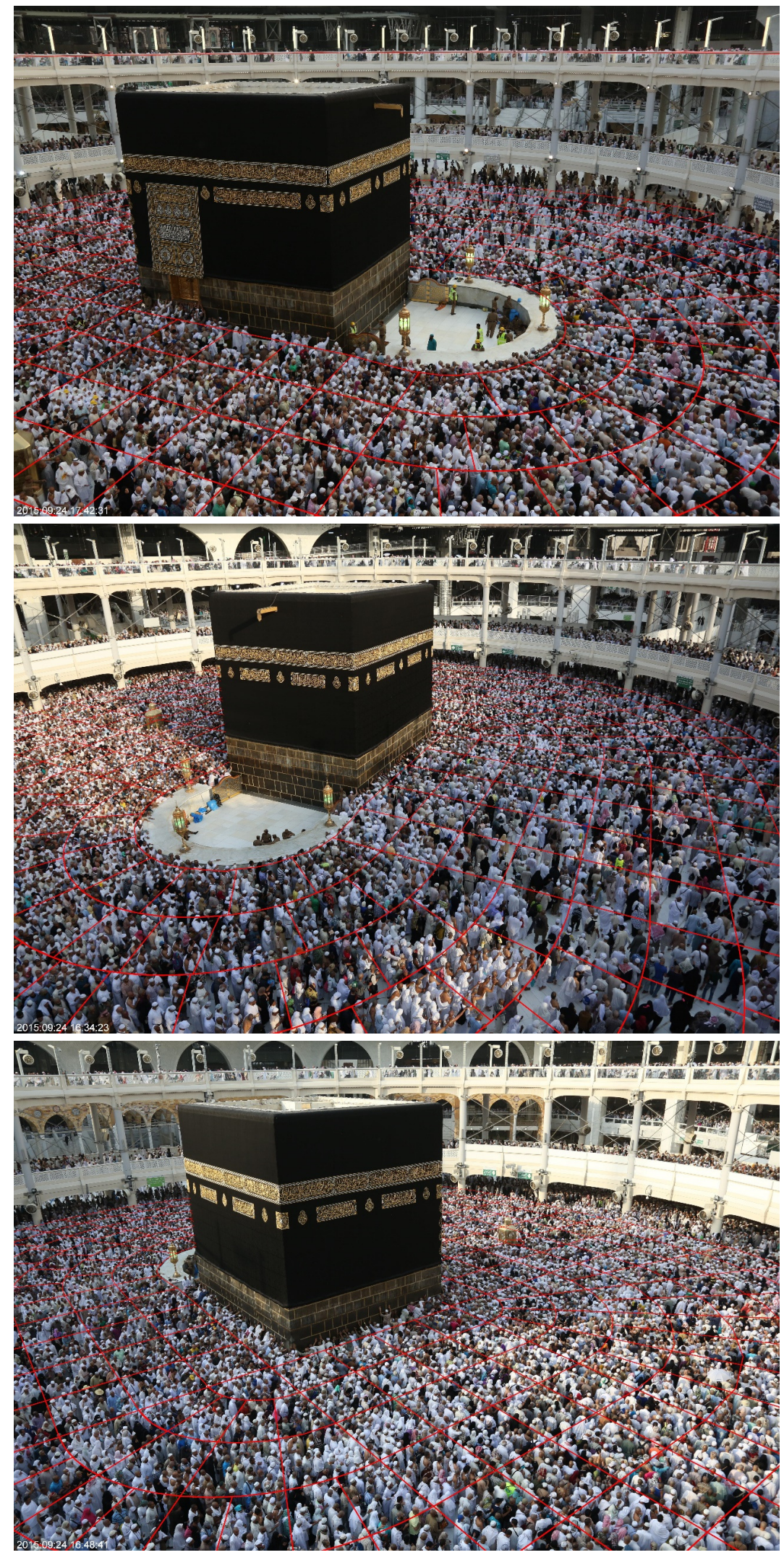

Figure 2 Typical image from film (positions 1,2,3) 


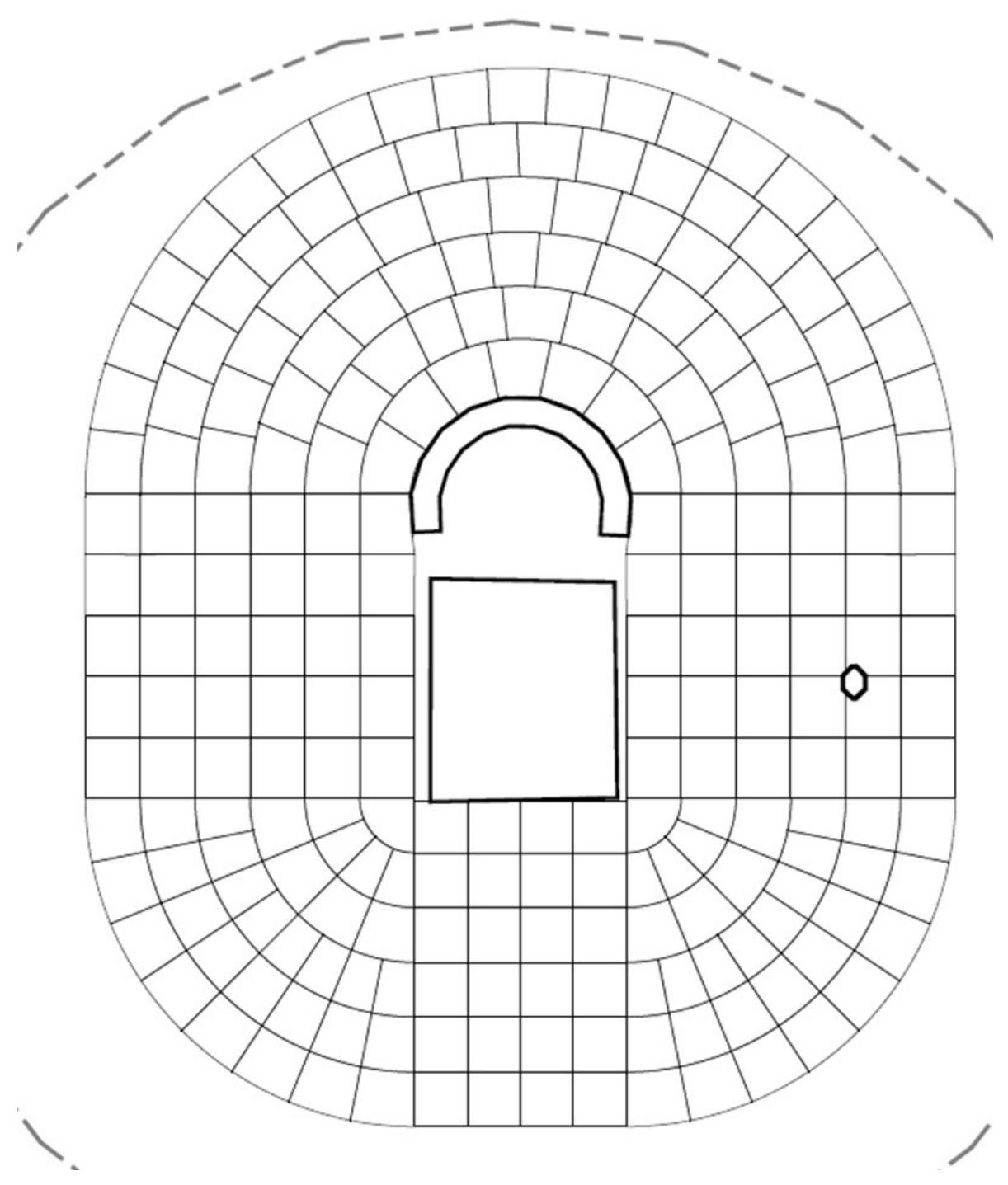

Figure 3 Zones of $10 \mathrm{~m}^{2}$ in area considered

used to film the pilgrims at 3 and 6 frames per second [fps] on the 2nd and 4th of October of 2014 (8th and 10th Dhu al-Hijjah), as well as the 24th of September of 2015. A few data points were also collected from videos at $24 \mathrm{fps}$. The cameras were placed in three positions on the upper ring level, approximately $15.1 \mathrm{~m}$ above the Sahn floor. The floor plan of the zone of observation, together with the subdivision into areas, can be seen in Fig. 1. The three camera locations may be inferred from Fig. 2. The crowds were filmed from three fixed positions during approximately one hour. During the Hajj of 2014, only two zones were covered. Fortunately, these were the zones of highest density. 


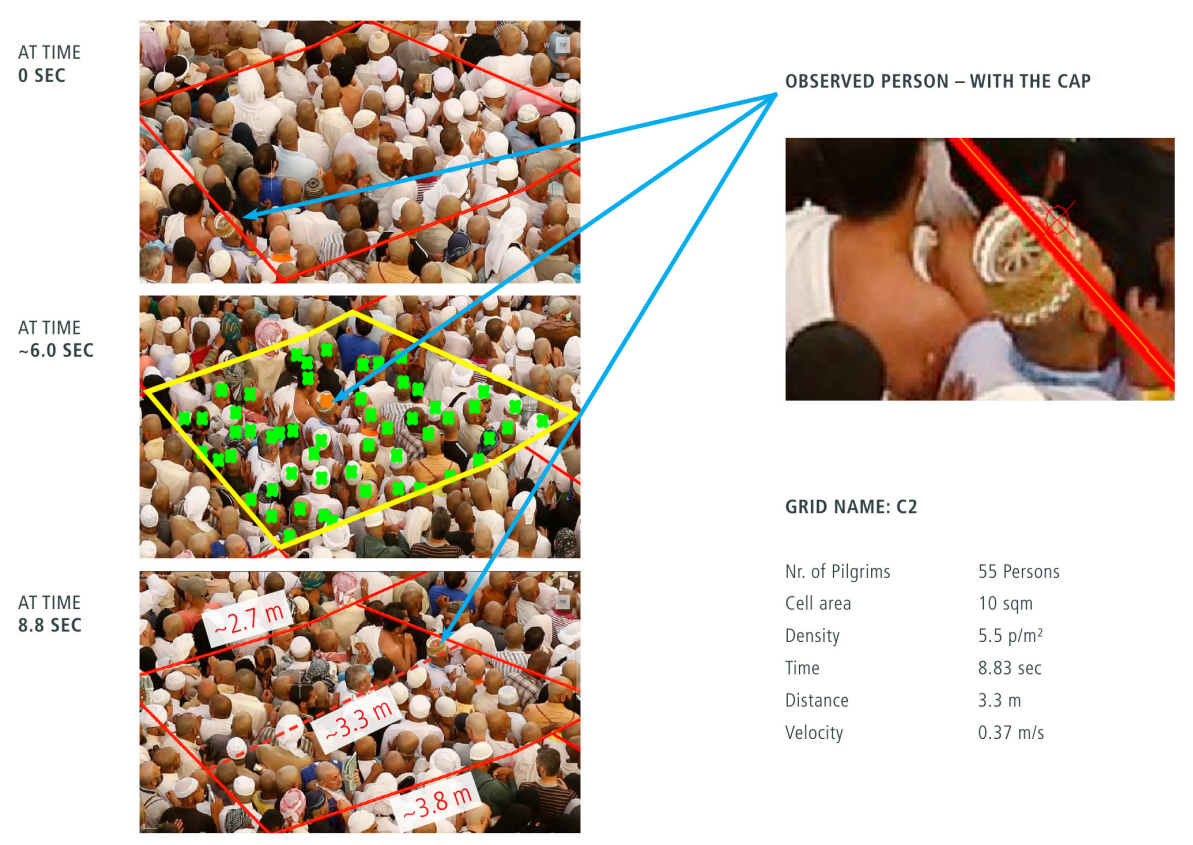

Figure 4 Evaluation of density and velocity

\section{Measurements and Data Processing}

In order to process the video images, the area of interest was subdivided into cells of $10 \mathrm{~m}^{2}$ using AutoCad (Fig. 3). Note that as it is impossible to always guarantee cells of uniform size when tesselating an arbitrary geometry, some cells are smaller that $10 \mathrm{~m}^{2}$. This deviation was taken into account when evaluating the results. By matching a highly accurate 3D model of the Al-Kaaba mosque generated at SLR for architectural and engineering purposes, and using GPS coordinates and survey data, the AutoCad lines were positioned at the head height of $1.5 \mathrm{~m}$. These grids were then overlaid to the original photos, yielding the final photos used for evaluation (see Fig. 2).

A number of automatic counting procedures were tried. Additionally, several pictures were sent to research groups and companies considered experts in the field. None of these attempts yielded any useful results. The decision was then made to perform the counts manually at SLR.

For any particular instant in time and any particular cell, a pilgrim whose transit was approximately through the middle of the cell and who could be easily followed (either because of height, garment colour or any other feature that made it easy to observe) was chosen and followed. The frame numbers when entering and exiting the cell were recorded, and from this data the average velocity during the transit across the cell was computed. The total number of pilgrims in the cell were counted when this 'marker pilgrim' was in the center of the cell, and this value was used to compute the density. A typical case is shown in Fig. 4. 


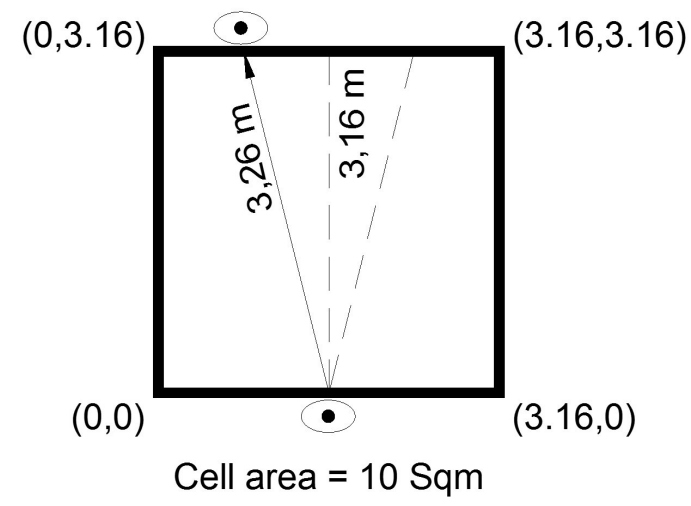

Figure 5 Estimation of error in transit length

The potential measurements errors were bounded as follows:

a) Velocity: The average velocity recorded is given by:

$$
v_{a v}=\frac{L}{t_{T}}
$$

where the average length of a cell is approximately $L=3 \mathrm{~m}$, and $t_{T}$ denotes the transit time. Given that trajectories that differed too much from the center of the cell were discarded, and the very accurate information provided by the CAD data together with the high resolution of the photos, the maximum relative error for $L$ was estimated to be of $\varepsilon_{L}=3.2 \%$ (Fig. 5).

The errors in the transit time amount, on average, to no more than one frame. The relative error in the transit time is given by:

$$
\varepsilon_{t}=\frac{\Delta t}{t_{T}}
$$

where $\Delta t$ is the time duration of one frame and $t_{T}$ the transit time. The average and maximum relative errors in velocity are then given by:

$$
\varepsilon_{v}^{\text {ave }}=\sqrt{\varepsilon_{L}^{2}+\varepsilon_{t}^{2}}, \varepsilon_{v}^{\max }=\frac{\varepsilon_{t}+\varepsilon_{L}}{1-\varepsilon_{L}} .
$$

The ratio of $t_{T} / \Delta t$, which determines the error in transit time, depends on the average velocity and has been tabulated in Tab. 1 for both framerates used.

One can see that for the interesting regime of $v_{a v}<0.4 \mathrm{~m} / \mathrm{s}$ the maximum relative error is below $8 \%$ even for the lower framerate, and below $6.0 \%$ for the higher framerate. The root-mean-square and average errors are, of course, much lower.

b) Density: The (instantaneous) density is given by:

$$
\rho=\frac{N_{p}}{A},
$$

where $N_{p}$ is the number of pedestrians in the cell, and $A$ the area of the cell. As before, we consider the errors in area size to be very small. On the other hand, counting pedes- 


\begin{tabular}{rrrrr}
\hline$L[\mathrm{~m}]$ & $v_{a v}[\mathrm{~m} / \mathrm{s}]$ & $t_{T}[\mathrm{~s}]$ & $\varepsilon_{3}[\%]$ & $\varepsilon_{6}[\%]$ \\
\hline 3.00 & 1.00 & 3.00 & 16.10 & 9.27 \\
3.00 & 0.80 & 3.75 & 13.26 & 8.00 \\
3.00 & 0.60 & 5.00 & 10.57 & 6.75 \\
3.00 & 0.40 & 7.50 & 8.00 & 5.54 \\
3.00 & 0.20 & 15.00 & 5.54 & 4.36 \\
3.00 & 0.10 & 30.00 & 4.35 & 3.77 \\
\hline
\end{tabular}

Table 1 Maximum percentage errors for velocity measurements

\begin{tabular}{rrr}
\hline$\rho\left[\mathrm{p} / \mathrm{m}^{2}\right]$ & $N_{p}[\mathrm{p}]$ & $\varepsilon_{\rho}[\%]$ \\
\hline 1 & 10 & 10.00 \\
2 & 20 & 5.00 \\
4 & 40 & 2.50 \\
6 & 60 & 1.67 \\
8 & 80 & 1.25 \\
10 & 100 & 1.00 \\
\hline
\end{tabular}

Table 2 Percentage errors for density measurements

trians in cells was not always an easy and straightforward matter: smaller pedestrians are sometimes hidden by taller ones, and pedestrians that move close to the border of a cell may or may not be counted as inside. For the first class of problems having video footage proved invaluable: pedestrians could be tracked over several seconds, minimizing the errors due to occlusion. Errors for the 'border pedestrians' were minimized by having two evaluators look at the same set of photos, and deciding whether to consider pedestrians in or out. The errors in density are given by:

$$
\varepsilon_{\rho}=\frac{{ }_{-}^{+} \Delta N_{p}}{N_{p}},
$$

where $\Delta N_{p}$ is the error in counting pedestrians. Realistically, we can assume a counting error of one pedestrian per zone $\left(\Delta N_{p}=1\right)$. The relative density errors have been tabulated in Tab. 2 as a function of density.

One can see that for the interesting regime of $\rho>4 \mathrm{p} / \mathrm{m}^{2}$ the relative error is below $2.5 \%$, and decreases even further with increasing density.

c) Instantaneous vs. Average Density: The data recorded combined an instantaneous density (i.e. when the pedestrian was in the middle of the cell) with an average velocity (i.e. length of trajectory vs. time required). This could potentially lead to errors and inconsistencies if the variation of density is large during the passage of the pedestrian across the cell. In order to quantify this error, a number of cells were analyzed in depth, recording the variation in the number of pedestrians in the cell during the time of traversal. The results are summarized in Tab. 3. One can observe that, as expected, the relative error 

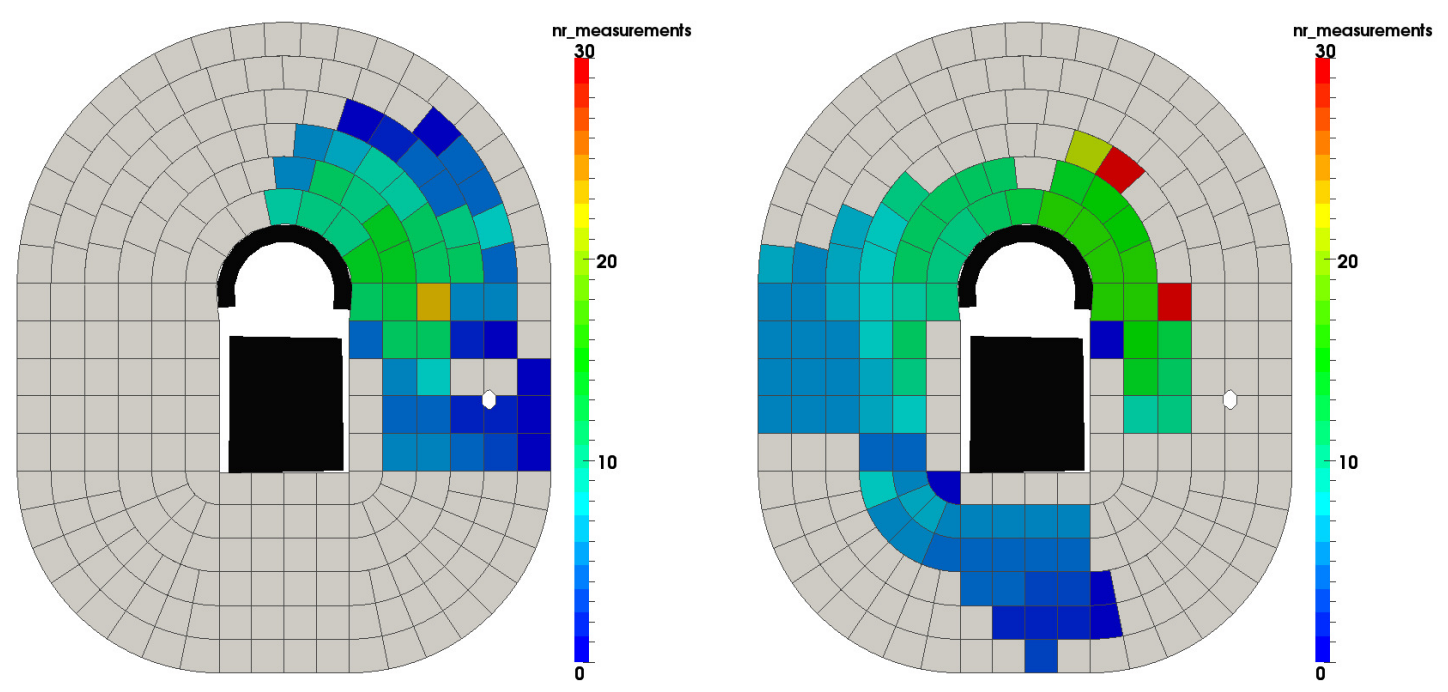

Figure 6 Number of measurements (left: Hajj 2014, right: Hajj 2015)

decreases with densities. At low densities, pedestrians can freely 'enter and exit' the cells, leading to higher relative errors, while at high densities this freedom is restricted and the variations are very small.

d) Flux: The flux or intensity is given by $f=\rho \cdot v$. This implies that the relative errors in flux are given by $\varepsilon_{f, r}=\sqrt{\varepsilon_{\rho, r}^{2}+\varepsilon_{v, r}^{2}}$, where,$r$ implies relative error. Note that in particular for the high density regime the errors in flux are mainly determined by the error in velocity and instantaneous vs average densities (both of which were bounded by $\left.\varepsilon_{v, r}<5 \%\right)$, implying that in this regime $\left(\rho>4 \mathrm{p} / \mathrm{m}^{2}\right)$ the relative error in fluxes is below $\varepsilon_{f, r}<5 \%$.

A total of 1,054 measurements (338 for the Hajj of 2014, 716 for the Hajj of 2015) were taken in this way. This corresponded to a total of 42,256 individual high resolution time-lapsed photos at $3 \mathrm{fps}$ and $6 \mathrm{fps}$ as well as videos at $25 \mathrm{fps}$. Approximately $10 \mathrm{~s}$ of frame data were used per data point. These photos and videos were taken over the span of 3,900 s for the Hajj of 2014 and 9,700 s for the Hajj of 2015. The number of measurements per cell is shown in Fig. 6.

One can see that certain zones, marked in grey, were not used for counting. There are several reasons for this. In the region close to the Maqqam Ibrahim, pilgrims stop to pray, so that this is not indicative of pedestrian flow. The same happens in the 3 zones at the

\begin{tabular}{rr}
\hline$\rho\left[\mathrm{p} / \mathrm{m}^{2}\right]$ & $\varepsilon_{\rho}[\%]$ \\
\hline 3 & 20.00 \\
4 & 5.00 \\
5 & 4.00 \\
6 & 3.40 \\
\hline
\end{tabular}

Table 3 Percentage errors of instantaneous vs average density 

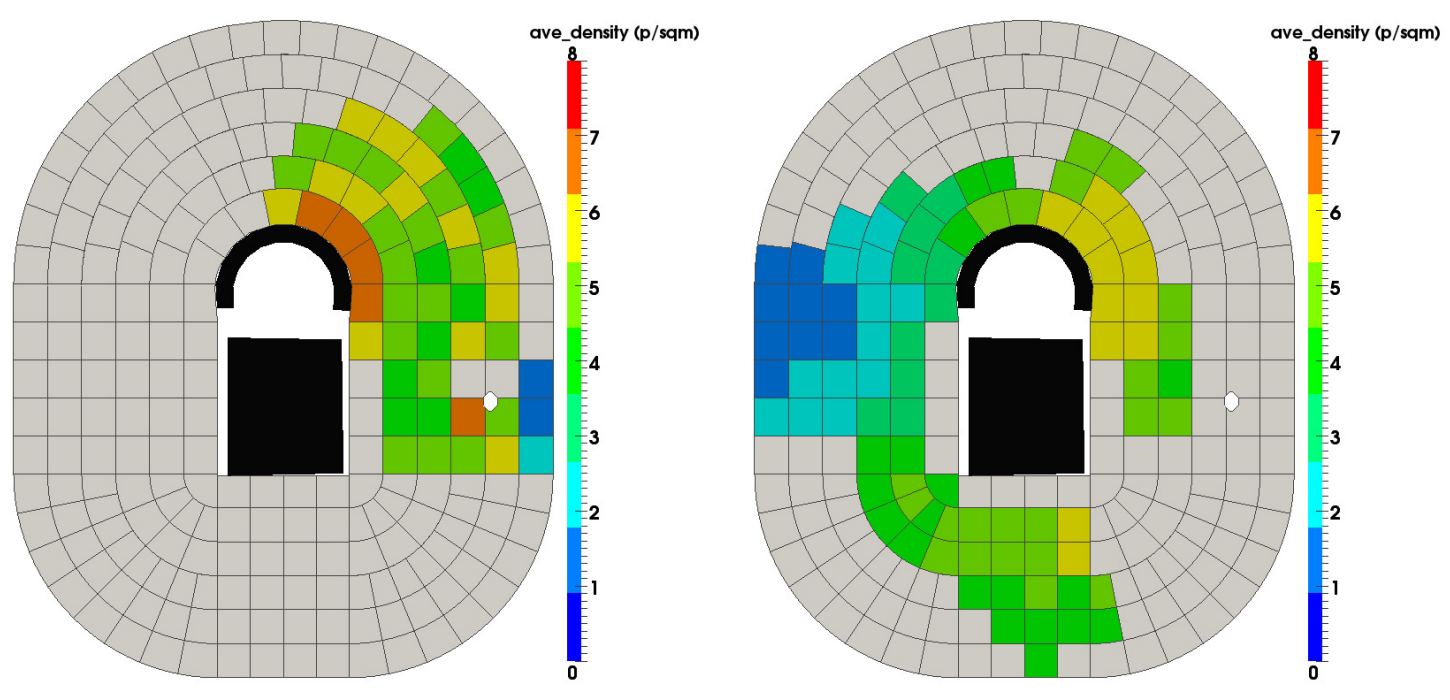

Figure 7 Average density (left: Hajj 2014, right: Hajj 2015)

bottom left that are adjacent to the Kaaba: pilgrims there stop or try to get to the black stone, so that again any measurement taken there would not be indicative of pedestrian flow. The zones at the top were not considered as the image distortion and observation angle and distance were deemed too large for accurate evaluations. The average and maximum values of density, velocity and flux were evaluated for the zones in which several countings were performed.

\section{Results Obtained}

The results obtained have been compiled according to zones, and are displayed in Fig. 710. Finally, all the data recorded was cast in the 'fundamental diagram' format as shown in Fig. 13, and compared to the curves published by [4] and [3]. Note that the velocity in the high density region does not vanish, leading to fluxes that exceed $f=3.5 \mathrm{p} / \mathrm{m} / \mathrm{s}$, much higher than previously reported. These results surprised the authors. Therefore, these areas were evaluated repeatedly and checked for errors. However, they held up to scrutiny, and are considered to be correct. Fig. 13 also provide the measurement times. Asr is the afternoon prayer time, Maghrib the sunset and Isha the evening prayer. We remark that the 2 nd of October 2014 was the so-called 'Day of Arafat' - implying that the volume of pedestrians was very low in comparison to the other (so-called 'Eid') dates.

\section{Discussion}

The first impression, especially for observers living in western cultures, is the very high average density $\left(\rho_{a v}=O(8) \mathrm{p} / \mathrm{m}^{2}\right)$, let alone maximum density $\left(\rho_{\max }=O(8-10) \mathrm{p} / \mathrm{m}^{2}\right)$ observed at the upper right part of the Kaaba, where the semi-circle begins. Even more surprising were the velocities, which were of the order of $v_{a v}=O(0.4) \mathrm{m} / \mathrm{s}$ ) leading to 


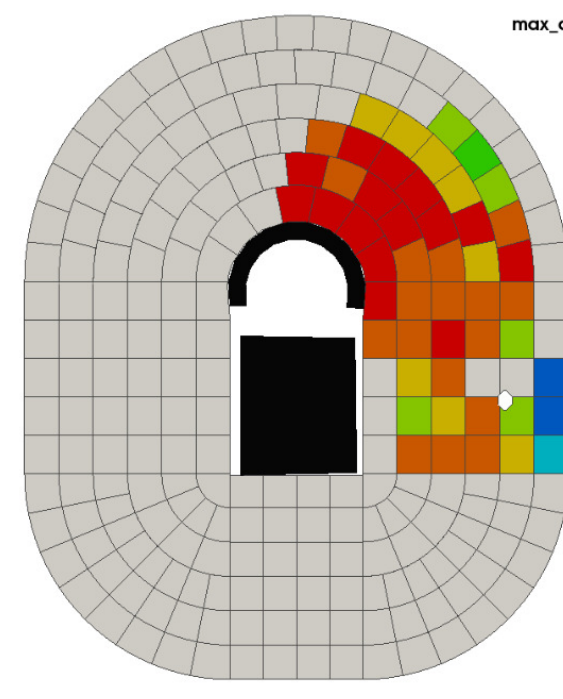

TAWAF : Hajj 2014

Max Density

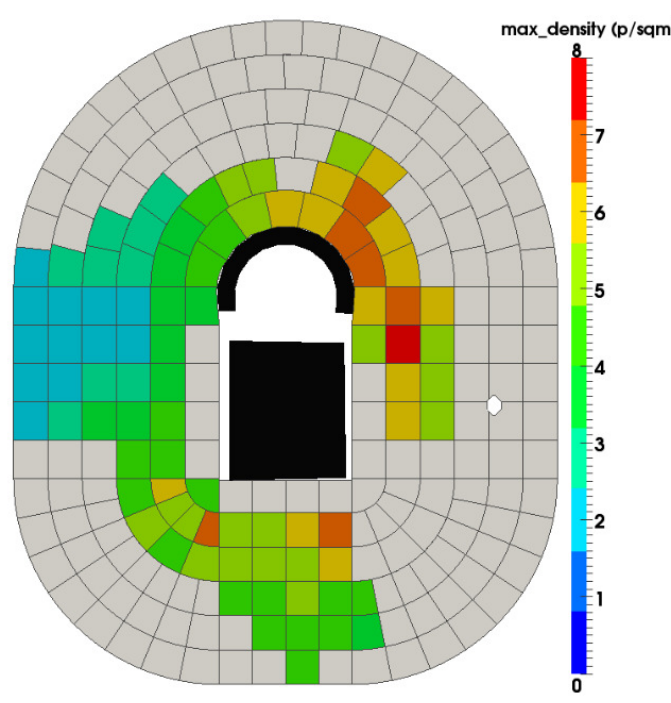

TAWAF : Hajj 2015

Max Density

Figure 8 Maximum density (left: Hajj 2014, right: Hajj 2015)
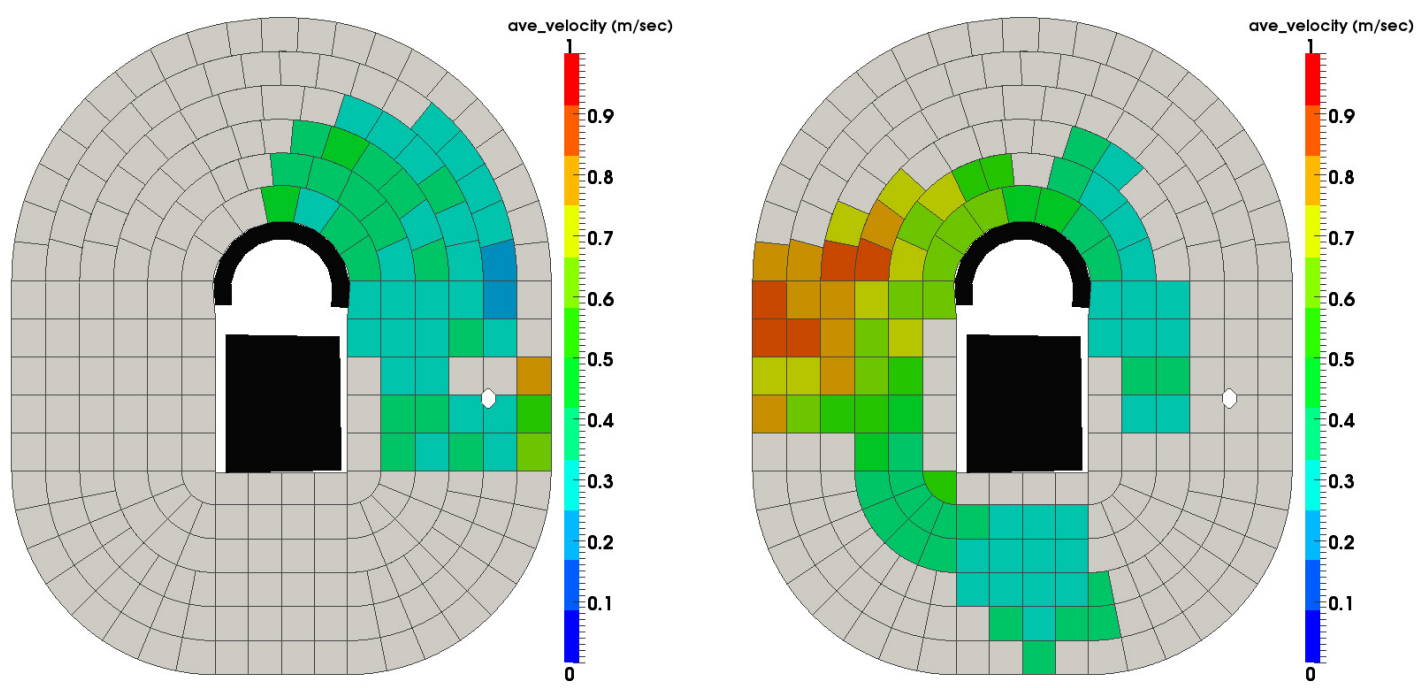

Figure 9 Average velocity (left: Hajj 2014, right: Hajj 2015) 

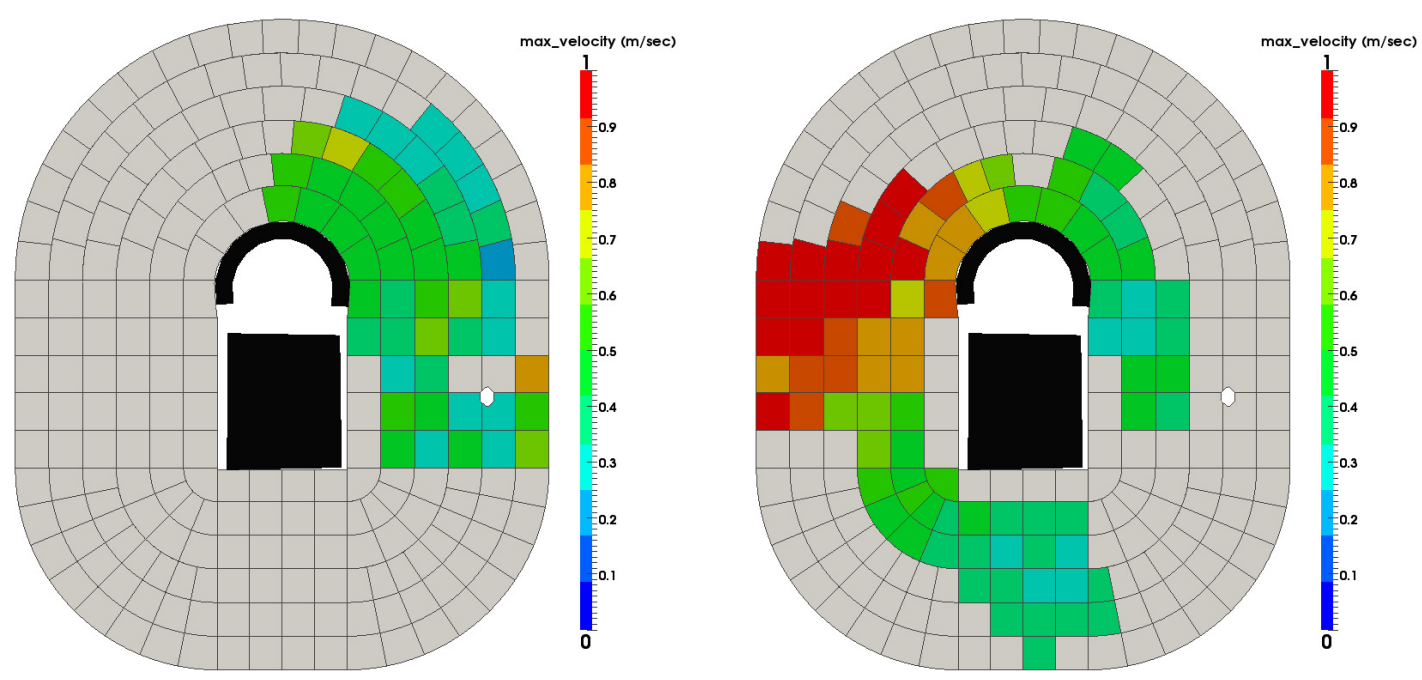

Figure 10 Maximum velocity (left: Hajj 2014, right: Hajj 2015)

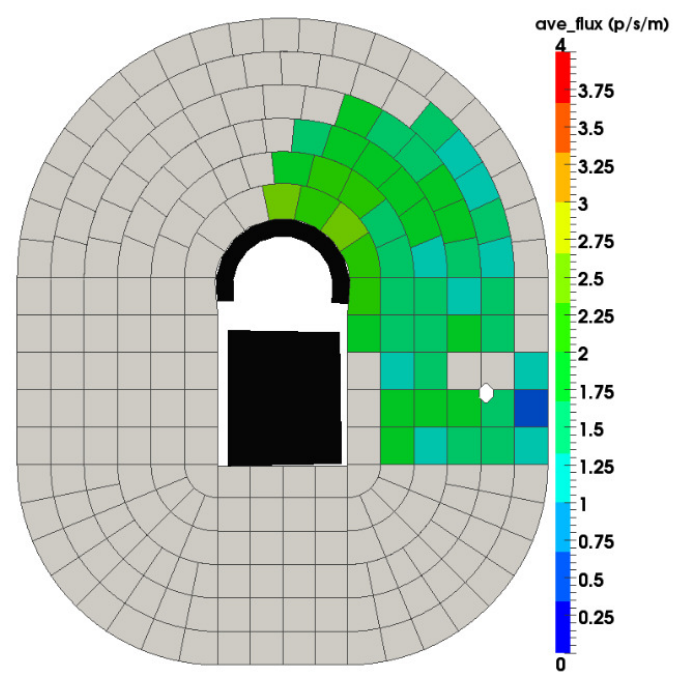

TAWAF : Hajj 2014

Average Flux

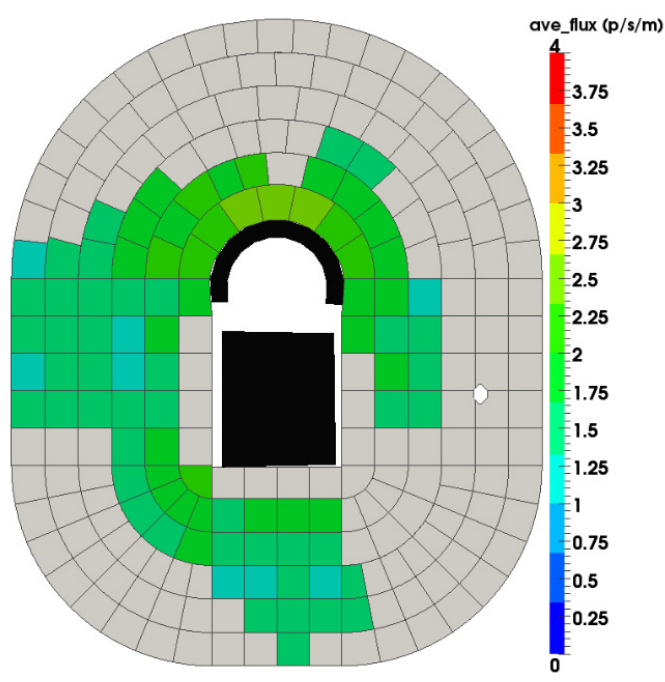

TAWAF : Hajj 2015

Average Flux

Figure 11 Average flux (left: Hajj 2014, right: Hajj 2015) 

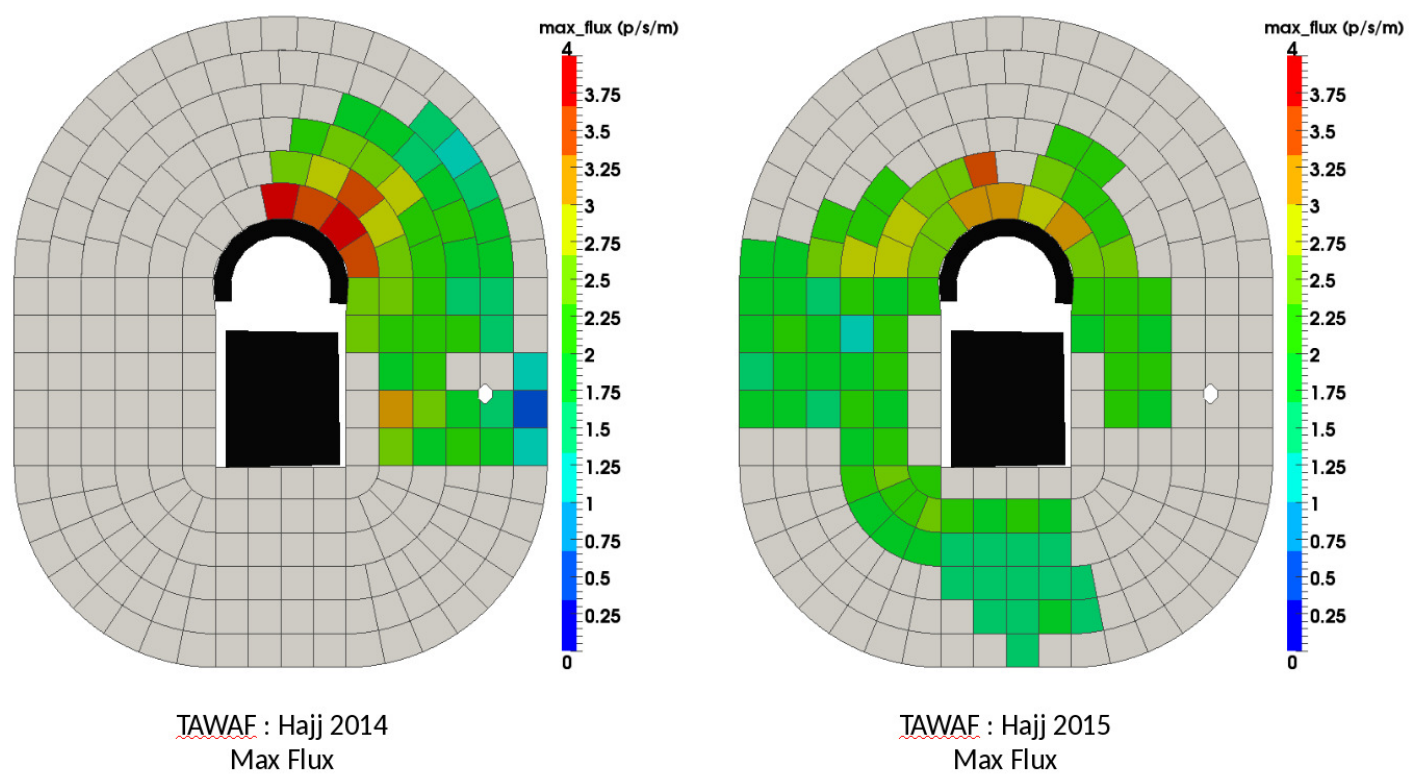

Figure 12 Maximum flux (left: Hajj 2014, right: Hajj 2015)

average fluxes exceeding $f=3.2 \mathrm{p} / \mathrm{m} / \mathrm{s}$. In this region, the maximum density for the first 3 'rings of zones' exceeds $\rho_{\max }=O(8) \mathrm{p} / \mathrm{m}^{2}$ while the velocities are still above $v=O(0.4) \mathrm{m} / \mathrm{s}$. It is doubtful that such high densities and velocities can be maintained for long corridors, but the present 'pedestrian corridor' that forms in this region spans $O(20) \mathrm{m}$ and is wider than $O(10) \mathrm{m}$, i.e. not inconsiderable. Pilgrims know that once they complete the semi-circle, the density drops considerably to bearable levels of $\rho=$ $O(4-6) \mathrm{p} / \mathrm{m}^{2}$. It may be that they 'endure' this high density and are disciplined enough not to despair and break into disorder. Fig. 13 indicate that the average velocities and fluxes recorded in the regions with densities $1.0 \leq \rho \leq 4.0 \mathrm{p} / \mathrm{m}^{2}$ on the 2 nd of October 2014 (Day of Arafat) lie below the Predtetschenski and Milinski curves, leading to fluxes that are $30 \%-50 \%$ below the ones reported in [4]. This trend is reversed on the 4th of October 2014 (Eid day) and 24th of September 2015 (Eid day), where the resulting fluxes are $30 \%-50 \%$ higher. For the regions above $\rho=O(7) \mathrm{p} / \mathrm{m}^{2}$ the fluxes that are almost twice as high as those reported in [4]. The monotonic increase in fluxes with density may also help to explain why in this region hardly any accidents have ever been reported. On the other hand, this casts serious doubts on the ability of 'mean' or 'average' curves such as those found in [4] to be used safely when designing for capacity in complex environments such as the ones measured.

Another surprising finding is the very high spread of data across the whole density regime. Previously, conventional wisdom expected a narrowing in the spread of velocities and fluxes in the high density regime. While a certain narrowing in the spread of velocities is observed at high densities, due to the multiplication with density no such narrowing could be observed for the fluxes. 

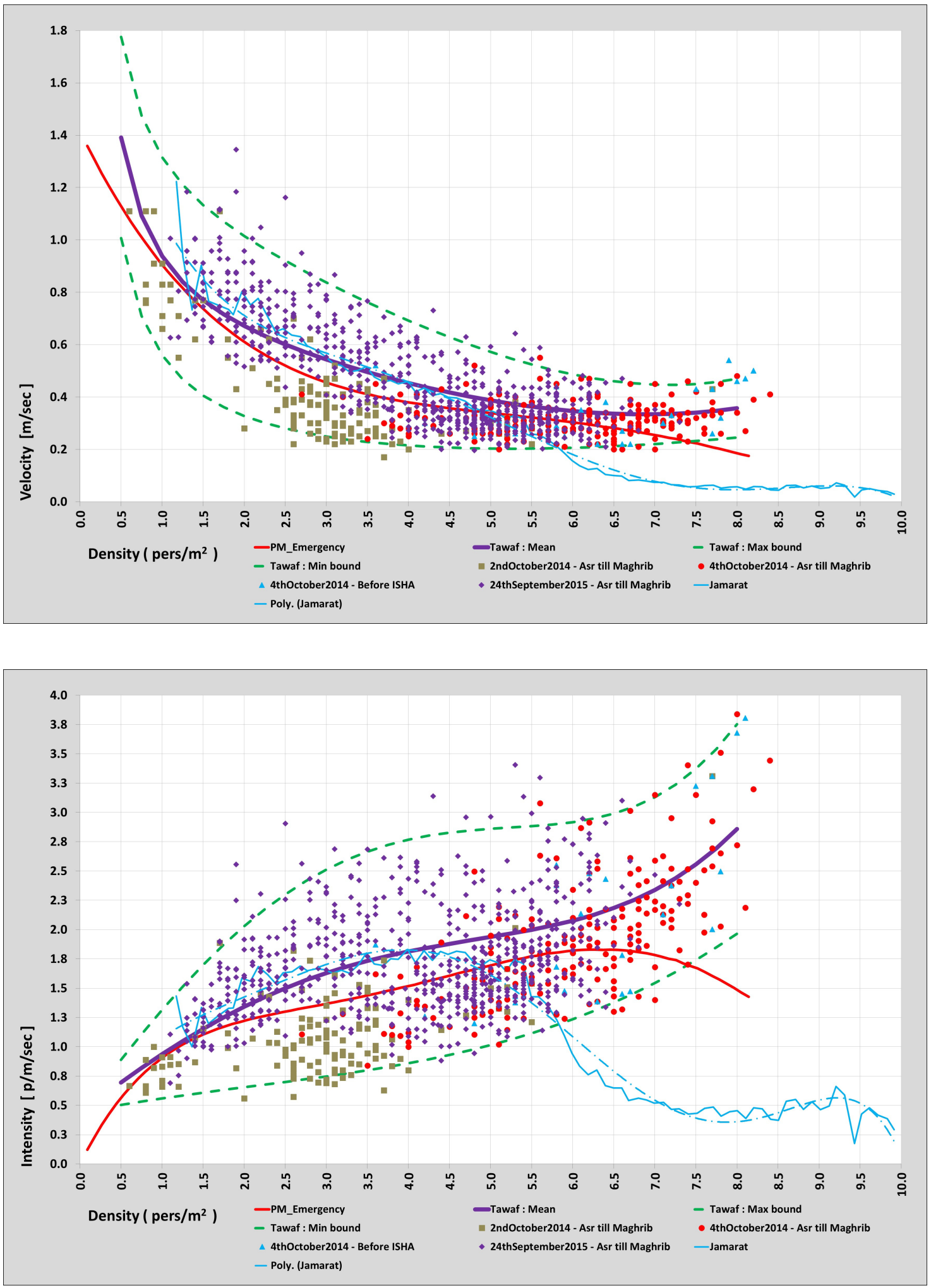

Figure 13 Fundamental diagram: velocity and flux as a function of density (detail, Hajj 2014/2015 data) 


\section{Conclusions}

Motivated by the desire to understand the motion patterns observed in the heavily crowded Mekka Sahn area as a basis for future architectural design campaigns, an experimental campaign was undertaken during the Hajj pigrimages of 2014 and 2015 to measure pedestrian densities and velocities in the region close to the Kaaba. High resolution video and photographs were used. The space was divided into areas of $10 \mathrm{~m}^{2}$. The pedestrians were counted, and the velocity measured from video clips. A total of 1,057 individual measurements were taken on three days. In light of published standard 'fundamental diagrams', the results were surprising, showing an increase in velocity in the very high density region, implying in turn an increase of the flux. The flux values obtained in regions with densities higher than $\rho=O(8) \mathrm{p} / \mathrm{m}^{2}$ exceeded $f=3.2 \mathrm{p} / \mathrm{m} / \mathrm{s}$, far above any reported flux values to date.

The measurements also indicate that the average velocities and fluxes recorded in the regions with densities $1.0 \leq \rho \leq 4.0 \mathrm{p} / \mathrm{m}^{2}$ can deviate as much as $50 \%$ from 'standard curves' reported in [4]. For the regions above $\rho=O(7) \mathrm{p} / \mathrm{m}^{2}$ the fluxes that are almost twice as high as those reported in [4]. One should add, though, that the data leading to the 'standard curves' obtained by Predtetschenski and Milinski [4], was mainly for straight passages and also showed considerable spread.

This may cast doubt about the 'universality' of fundamental diagrams found in the literature, and, more importantly, about their indiscriminate application to design for and operation of heavily crowded events. While scientists may value universality and generality, the discrepancy with measurements taken in other venues and situations may indicate that fundamental diagrams depend strongly on the type of motion and on the nature of the event. Perhaps one should classify them according to motion types, such as 'concerted', 'self-organized', 'intention-based', 'marching columns', etc. Alternatively, classes of heavily crowded events should be charted and published, such as religious, sports, cultural, political, festival, etc..

Another important conclusion for planners and designers is that our knowledge of pedestrian motion is still far from complete, making case-to-case measurements such as the ones reported here an imperative ingredient of any architectural endeavour.

\section{References}

[1] Fruin, J.J.: Pedestrian Planning and Design; New York: Metropolitan Association of Urban Designers and Environmental Planners (1971)

[2] Gdoura, M.K., Löhner, R., Haug, E., Gawenat, B.: On the Influence of Columns in Densely Populated Corridors; in Proc. Conf. Pedestrian and Evacuation Dynamics 2014 (PED2014), Delft, October (2014)

[3] Helbing, D., Johansson, A., Al-Abideen, H.Z.: The Dynamics of Crowd Disasters: An Empirical Study; Phys. Rev. E 75, 046109 (2007) 
[4] Predtetschenski, W.M., Milinski, A.I.: Personenströme in Gebäuden - Berechnungsmethoden für die Projektierung; Verlaggesellschaft Rudolf Müller, KölnBraunsfeld (1971).

[5] Schadschneider, A., Klingsch, W. , Klüpfel, H., Kretz, T., Rogsch, C., Seyfried, A.: Evacuation Dynamics: Empirical Results, Modeling and Applications; pp. 3142-3176 in Encyclopedia of Complexity and Systems Science (R.A. Meyers ed.), Springer, New York (2009)

[6] Seyfried, A., Steffen, B., Klingsch, W., Boltes, M.: The Fundamental Diagram of Pedestrian Movement Revisited; J. Stat. Mech. P10002, arXiv:physics/0506170 [physics.soc-ph] (2005)

[7] Seyfried, A., Portz, A., Schadschneider, A.: Phase Coexistence in Congested States of Pedestrian Dynamics, arXiv:1006.3546 [physics.soc-ph] ACRI2010 - Workshop C\&CS (2010)

[8] Virkler, M.R., Elayadath, S.: Pedestrian Speed-Flow-Density Relationships; Transportation Research Record 1438, 51-58, (1994)

[9] Weidmann, U.: Transporttechnik der Fussgänger, Schriftenreihe des IVT Nr. 90, 2. Ergänzte Auflage, ETH Zürich (1993)

[10] Zhang, J.: Pedestrian Fundamental Diagrams: Comparative Analysis of Experiments in Different Geometries, Schriften des Forschungszentrums Jülich, IAS Series Vol. 14 (2012) 